\title{
Le transfert du mercure (Hg) utilisé comme descripteur du fonctionnement hydrologique (échanges cours d'eau - nappe) dans la plaine alluviale du Rhin supérieur : impact des aménagements
}

\author{
U. Roeck 1 \\ M. Trémolières ${ }^{2}$ \\ A. Exinger 1 \\ R. Carbiener ${ }^{2}$
}

Mots clés : Fonctionnement hydrologique, champ d'inondation fonctionnel, bioindicateurs d'accumulation, échanges rivière-nappe, mercure, contamination de l'eau souterraine.

Des dosages de mercure $(\mathrm{Hg})$ ont été effectués dans des espèces de mousses aquatiques (bryophytes), Fontinalis antipyretica Hedw., Cinclidotus danubicus Schiffn. \& Baumg., Cinclidotus nigricans (Brid.) Wijk \& Marg. et Amblystegium riparium Hedw., prélevées dans le réseau hydrographique de la plaine rhénane d'Alsace (Rhin canalisé, son principal affluent l'Ill qui possède un champ d'inondation fonctionnel et dans le réseau des rivières phréatiques, Fig. 1).

Le choix des points de prélèvement a été basé sur les résultats obtenus lors des études antérieures utilisant les méthodes des traceurs hydrologiques $\left(\mathrm{Cl}^{-}\right)$, et des analyses physico-chimiques de l'eau $\left(\mathrm{NO}_{3}{ }^{-}, \mathrm{PO}_{4}{ }^{3-}, \mathrm{NH}_{4}{ }^{+}\right)$et la méthode de bioindication de la qualité de l'eau basée sur les groupements végétaux.

Des variations caractéristiques des teneurs mercurielles des mousses aquatiques ont été observées (Fig. 2). Des teneurs (moyennes annuelles) plus élevées (allant jusqu'à $0,29 \mu \mathrm{g} \mathrm{Hg} \cdot \mathrm{g}^{-1}, \mathrm{~ms}$ ) relevées dans les cours d'eau phréatiques concernent surtout ceux situés à proximité du Rhin et visualisent des secteurs d'infiltration d'eau contaminée du fleuve canalisé vers sa nappe riveraine. Des zones de la nappe à faibles teneurs en mercure des mousses $\left(0,05 \mu \mathrm{g} \mathrm{Hg} . \mathrm{g}^{-1}\right.$, ms) représentent des secteurs de la nappe exempts de contamination mercurielle.

Les zones les plus vulnérables de la nappe phréatique rhénane sont les secteurs amont des usines hydroélectriques et les coudres du fleuve (Fig. 5), d'une part pour la pollution mercurielle « historique » du Rhin canalisé, et d'autre part pour des contaminations chroniques, surtout par des polluants persistants.

Au contraire du Rhin canalisé, la rivière Ill également fortement contaminée par le $\mathrm{Hg}$, dans la partie aval de son cours, ne fuit pas dans son lit mineur. Lors des inondations, cette rivière assure l'épuration des eaux de crue grâce au système de rétention « sol-végétation » et recharge ainsi-la nappe phréatique en eau d'excellente qualité : la végétation absorbe des nutriments et les colloïdes du sol (argiles, limons) adsorbent des cations et donc du mercure.

Les résultats de cette étude confirment l'impact de la canalisation du Rhin sur les échanges rivière - aquifère et l'importance de la préservation d'un champ d'inondation fonctionnel pour la qualité de l'eau des nappes alluviales.

Mercury (Hg) transfer as a descriptor of the hydrological functioning (water course - watertable exchange) of the upper Rhine flood plain : impact of river canalizations

Keywords : Hydrological functioning, functional floodplain, bioaccumulation, river-groundwater interaction, mercury, groundwater contamination.

Mercury ( $\mathrm{Hg}$ ) levels in 4 aquatic moss-species (Bryophytes) from the hydrographical system of the Alsatian floodplain (canalized Rhine, its main tributary, the Ill river with a functional floodplain, groundwater-fed stream network) were analyzed (Fig. 1.). The moss-species Fontinalis antipyretica Hedw., Cinclidotus danubicus Schiffn. \& Baumg., Cinclidotus nigricans (Brid.) Wijk \& Marg. and Amblystegium riparium Hedw. were found at various sites according to the characteristics of the river.

The choice of the sampling sites was based on results obtained in former studies (Carbiener \& Herrscher 1989, Carbiener \& Trémolières 1990, Trémolières et al. 1991, Eglin \& Robach 1992) using different methods of water quality investigation and river-aquifer interaction (hydrological tracer : $\mathrm{Cl}-$; physico-chemical parameters : $\left(\mathrm{NO}_{3}{ }^{-}, \mathrm{PO}_{4}{ }^{3-}, \mathrm{NH}_{4}{ }^{+}\right)$; phytosociological bioindication scale using river macrophyte communities).

1. Laboratoire d'Hydrologie ;

2. Laboratoire de Botanique et d'Ecologie végétale, CEREG, CNRS URA 95 U.E.R. de Sciences Pharmaceutiques, Université Louis Pasteur Strasbourg, 74, route du Rhin, 67401 Illkirch cedex, France.

Texte d'une communication effectuée dans le cadre du Colloque - Limnologie appliquée et application de la Limnologie - Besançon, Franche-Comté, 16-19 novembre 1992. 
Characteristic differences in $\mathrm{Hg}$ contents in the aquatic bryophytes from the groundwater-fed streams were found (Fig. 2). Higher annual average $\mathrm{Hg}$ contents in the bryophytes (up to $0,29 \mu \mathrm{g} \mathrm{Hg} . \mathrm{g}^{-1}$, dw) from the groundwater-fed streams reflected river seepage sectors which are all located close to the canalized Rhine.

Low level zones $\left(<0,05 \mu \mathrm{g} \mathrm{Hg} . \mathrm{g}^{-1} ; \mathrm{dw}\right)$ indicated non-contaminated and drained sectors of the alluvial groundwater table.

The most vulnerable sectors of the groundwater table close to the Rhine, not only with regard to the " historical » $\mathrm{Hg}$ pollution, but also for chronic contaminations (especially with persistent pollutants), are the sectors upstream from the hydroelectric power plants and the river bend (Fig. 5). The hydrostatic pressure of the watercolumn leads to the infiltration of contaminated river water through the gravel bed of the Rhine into its riparian aquifer.

By contrast with the canalized Rhine, the Ill river, also highly contaminated by mercury, does not show river seepage.

When it is in flood, this river imports high quality water to the aquifer. Its functional floodplain, the " soil-vegetationsystem ", purifies the floodwater : the vegetation absorbs nutrients and the soil colloïds (clay, limon) absorb cations, and thus mercury.

The results of this study confirm the impact of river canalization on river-aquifer exchange activities and thus the importance of « functional-floodplain-preservation » for alluvial groundwater-quality.

\section{Introduction}

Les travaux d'aménagements fluviaux perturbent considérablement le fonctionnement hydrologique et écologique des hydrosystèmes alluviaux, et augmentent leur vulnérabilité vis-à-vis de contaminations ponctuelles ou chroniques. En effet, la canalisation d'un cours d'eau peut profondément modifier la typologie de ce dernier, ainsi que les flux entre les différents sous-systèmes fonctionnels de l'hydrosystème (eau de surface - eau souterraine), mais aussi les flux à l'intérieur de ces derniers (Amoros et al. 1988, Ramade 1981, Haslam 1992, Davies et al. 1992).

La plaine alluviale du Rhin supérieur, du fait de l'homogénéité globale de l'aquifère (graviers carbonatés calciques fluvio-glaciaires), permet l'étude comparative des échanges entre les eaux de surface (Rhin canalisé, Ill) et leur nappe phréatique adjacente.

Cette étude vise (1) à vérifier si le mercure qui contamine fortement le Rhin et l'Ill, est transféré vers les nappes riveraines de ces deux cours d'eau au cours des échanges rivière-nappe et (2) à confirmer les résultats d'études antérieures prenant en compte les paramètres physico-chimiques de l'eau (Krause \& Carbiener 1975, BRGM/SGAL 1978), ou l'échelle de bioindication phytosociologique de la qualité de l'eau basée sur la présence/absence de groupements de macrophytes aquatiques (Ortscheit \& Carbiener 1987, Carbiener et al. 1988, Carbiener
\& Trémolières 1990, Eglin \& Robach 1992) ou encore des bioindicateurs d'accumulation poissons (Carbiener 1978). Les résultats de ces méthodes ont démontré le fonctionnement hydrologique différencié selon le degré d'aménagement d'une rivière, à savoir si elle est canalisée ou si elle possède un champs d'inondation fonctionnel. Il s'agit de localiser d'une part les apports de mercure du Rhin canalisé vers la nappe phréatique riveraine (Eglin \& Robach 1992, Trémolières et al. 1993) pour comprendre l'influence de la canalisation du fleuve sur sa nappe et d'autre part le rôle épurateur et protecteur d'un champ d'inondation fonctionnel, visà-vis d'eutrophisants et de micropolluants, pour une nappe phréatique riveraine (Sanchez-Perez 1992), comme par exemple celle de l'Ill (Trémolières et al. 1993, Roeck 1992).

\section{Matériel et méthodes}

\subsection{Description de la zone d'étude}

La zone d'étude est localisée dans la plaine alluviale du Rhin supérieur en Alsace aux caractéristiques hydro-géomorphologiques particulières (Carbiener 1983). Le fleuve coule dans le fossé tectonique rhénan, entre la Forêt Noire à l'est et les Vosges à l'ouest. Le Rhin postglaciaire a creusé son lit dans ses propres alluvions constituées de graviers grossiers, de galets et de sables atteignant, par endroit, jusqu'à $160 \mathrm{~m}$ d'épaisseur. Ces graviers constituent un des aquifères les plus importants 
à l'échelle européenne (Simler et al. 1979, ClootsHirch 1987). La perméabilité du gravier facilite le déplacement des eaux souterraines et favorise les échanges entre le Rhin et sa nappe riveraine. La nappe, souvent affleurante ou subaffleurante, est à l'origine d'une zone humide importante, le « Grand Ried Central d'Alsace » situé à environ $30 \mathrm{~km}$ au sud de Strasbourg dans l'ancien secteur des tresses et anastomoses du Rhin sauvage avant la rectification (19e siècle) et la canalisation de ce dernier réalisée au cours de ce siècle.

Cette zone humide est caractérisée par un réseau dense de cours d'eau qui correspond en grande partie à d'anciens bras du fleuve entièrement déconnectés de ce dernier depuis sa canalisation. Ces cours d'eau sont alimentés exclusivement ou quasi exclusivement par la nappe phréatique, d'où l'appelation « rivières phréatiques ». De ce fait, la qualité des eaux des rivières phréatiques réflète la qualité des eaux de nappe.

L'aménagement du Rhin supérieur qui a conduit à la mise en place d'une succession de biefs a supprimé la majeure partie du champ d'inondation du fleuve qui assurait, jusqu'à la canalisation, la recharge de la nappe phréatique en eau de bonne qualité (Carbiener 1978).

L'élévation du niveau d'eau du Rhin canalisé par rapport à celui de la nappe phréatique, surtout au niveau des bassins de rétention, favorise des infiltrations directes d'eau contaminée du fleuve appelées « filtrats rhénans » (Carbiener et al. 1988) vers la nappe phréatique riveraine.

\subsection{Application de la méthode des bryophytes aquatiques}

Au travers d'une pollution mercurielle grave mais déjà ancienne des eaux du Rhin et de l'Ill (Carbiener 1977, Carbiener 1978), ce travail étudie les mécanismes d'échange entre ces cours d'eau et leurs nappes riveraines. La contamination de l'eau souterraine par le mercure est visualisée par les teneurs en mercure $(\mathrm{Hg})$ des bryophytes aquatiques accumulateurs de métaux lourds (Mouvet 1986). L'identification des espèces a été réalisée à l'aide de la flore de Smith (1980).

Des prélèvements de mousses aquatiques ont été effectués tous les deux mois sur une période de 3 ans (1989 à 1991) dans les eaux de surface (Rhin et l'Ill) et dans les rivières phréatiques.
Le réseau des points de prélèvement (Fig. 1) est localisé dans l'ancien secteur des tresses et anastomoses du Rhin, aux échanges les plus actifs entre le fleuve et sa nappe riveraine (Carbiener et al. 1988) à hauteur du " Grand Ried Central d'Alsace ".

Le présent travail ne vise pas seulement à révéler le degré de pollution, mais à mettre en évidence le fonctionnement hydrologique des échanges cours d'eau-nappe et à étudier les variations spatiotemporelles éventuelles.

Les bryophytes aquatiques jouent le rôle de descripteur du fonctionnement hydrologique cours d'eau-nappe, analogue à l'utilisátion de groupements végétaux (Carbiener \& Ortscheit 1987, Klein 1988, Carbiener \& Trémolières 1990, Eglin \& Robach 1992, Robach et al. 1991), et animaux (Castella \& Amoros 1986), espèces, ou ensembles d'espèces animales hypogées et épigées (Lafont \& Durbec 1990) et, enfin, des sédiments (Rolland 1991).

Les mousses aquatiques sont prélevées à main nue, lavées à l'eau de la rivière et placées dans des papiers filtres dont l'absence de mercure a été vérifiée.

$\mathrm{Au}$ laboratoire, elles sont lavées (après, si nécessaire, réhumidification) et séchées à l'étuve $\left(60^{\circ} \mathrm{C}\right.$ pendant 24 heures). A la suite de la minéralisation $\left(48 \mathrm{~h}\right.$ à $\left.70^{\circ} \mathrm{C}\right)$ de 2 sous-échantillons de mousse pour chaque site et poür chaque campagne de prélèvement (en milieu acide, $\mathrm{HNO}_{3}$ conc. $/ \mathrm{H}_{2} \mathrm{SO}_{4}$ au demi et saturé en $\mathrm{KMnO}_{4} ; 1: 1$, le dosage du mercure est réalisé en AAS par la technique des « vapeurs froides » sur un appareil IL 357.

\subsection{Interprétation des résultats}

Selon l'hypothèse de travail posée, les sites au niveau desquels les bryophytes aquatiques présentent des teneurs élevées en mercure correspondent à des zones d'infiltration d'eau de rivière contaminée vers la nappe phréatique, tandis que les sites au niveau desquels les mousses révèlent des teneurs mercurielles faibles représentent des zones de drainage de la nappe phréatique ou des zones exemptes de contaminations mercurielles. L'interprétation des résultats est basée d'une part sur l'évolution temporelle et spatiale des teneurs en mercure en chaque point de prélèvement pour les différentes campagnes de prélèvement et d'autre part, sur les moyennes annuelles. Celles-ci permettent de visualiser la sectorisation de la nappe en intégrant les variations 


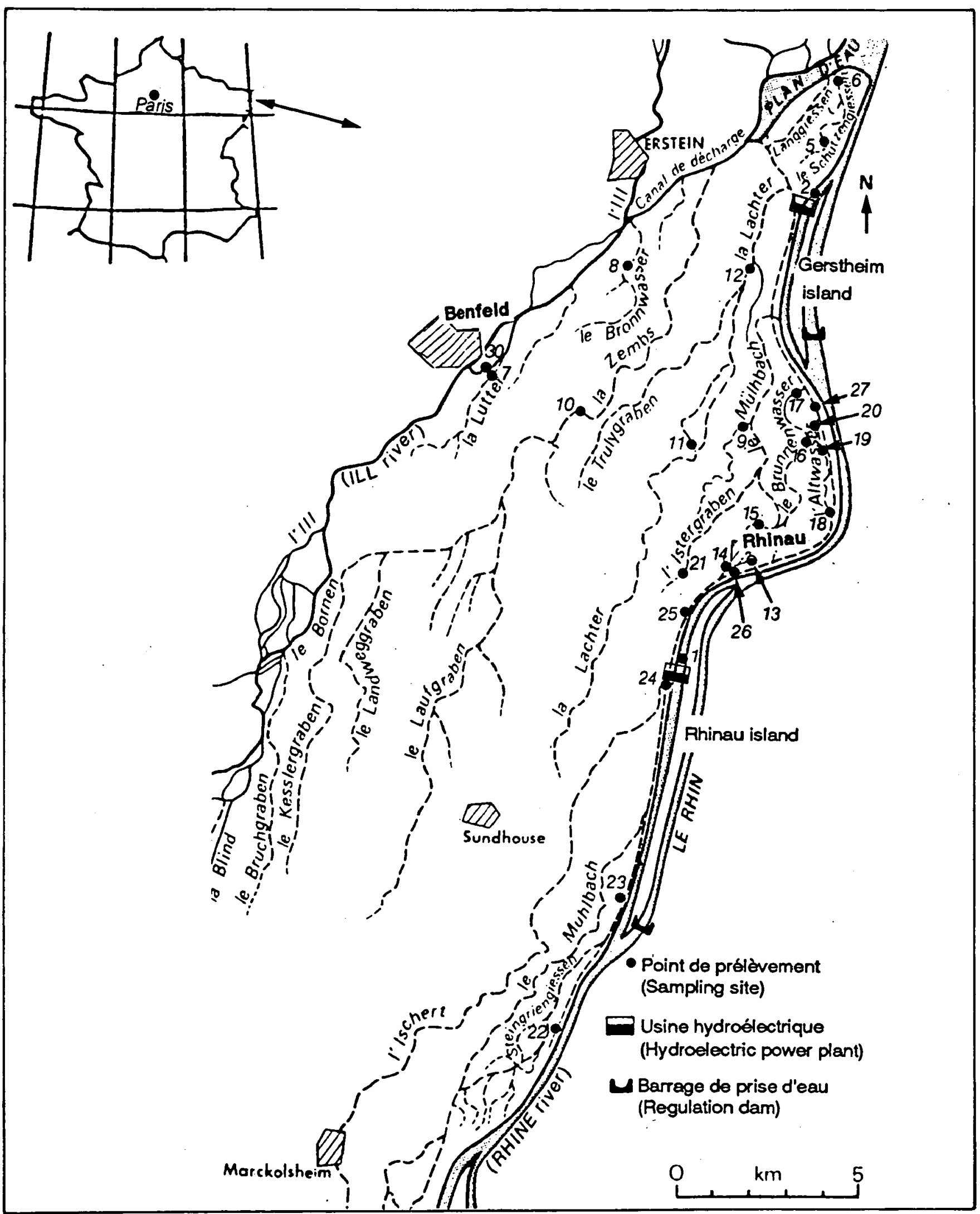

Fig. 1. Localisation de la zone d'étude et des points de prélèvement pour les bryophytes' aquatiques.

Fig. 1. Location of the study area and the sampling points for the aquatic bryophytes. 
saisonnières des teneurs en $\mathrm{Hg}$ des mousses aquatiques. Sur la base des moyennes annuelles nous avons défini des « secteurs de contamination " correspondant aux classes de concentrations suivantes : $<0,1$ $\mu \mathrm{g} \mathrm{g}^{-1}, 0,1$ à $0,2 \mu \mathrm{g} \mathrm{g}^{-1}$ et $>0,2 \mu \mathrm{g} \mathrm{g}^{-1}$.

\section{Résultats}

\subsection{Etude préliminaire}

L'étude préliminaire réalisée en 1989 portait sur les teneurs en mercure des mousses aquatiques des 6 campagnes de prélèvement sur une vingtaine de points de prélèvement dans le Rhin, l'Ill et dans les rivières phréatiques. De plus, pour la campagne de prélèvement du mois de mars, une recherche systématique de 12 éléments $(\mathrm{Cd}, \mathrm{Pb}, \mathrm{As}, \mathrm{Se}, \mathrm{Cr}, \mathrm{Ni}, \mathrm{Zn}$, $\mathrm{Sn}, \mathrm{Li}, \mathrm{Be}, \mathrm{B}$ et $\mathrm{Cu}$ ) dans les bryophytes aquatiques a été effectuée. Les résultats du screening ont révélé, en comparaison avec les valeurs de référence standard de Mouvet (1986), le niveau de contamination métallique suivant : les teneurs en $\mathrm{Cd}$, As, Ni et $\mathrm{Zn}$ ne dépassent en aucun point de prélèvement la valeur de référence standard pour un site exempt de pollution métallique (Roeck 1989). Seul un point de prélèvement montre une teneur élevée pour le $\mathrm{Pb}$ (facteur 3,2 par rapport à la valeur de référence). Deux points $(7,30)$ révèlent une contamination par le $\mathrm{Cr}$ (facteur 2,9 par rapport à la valeur de référence). Les teneurs en $\mathrm{Cu}$ montrent des variations spatiales allant jusqu'au facteur 4 (13) par rapport à la valeur de référence standard et jusqu'au facteur 10, « Facteur de contamination » FC (Mouvet 1986), par rapport au bruit de fond naturel de la zone d'étude (9).

Les teneurs des bryophytes aquatiques en $\mathrm{Hg}$, par contre, atteignaient des concentrations jusqu'à 8 fois plus fortes que celle de la valeur de référence standard et indiquaient une pollution certaine dans certains secteurs de la nappe phréatique. Sept points de prélèvement parmi les 20 ont présenté des teneurs supérieures d'un facteur 3 par rapport à la valeur de référence indiquant une contamination mercurielle certaine de l'eau souterraine.

\subsection{Contamination mercurielle}

Les résultats de l'étude préliminaire nous ont conduit à réaliser une étude plus approfondie des échanges Rhin-nappe phréatique, Ill-nappe phréatique basée sur le transfert du $\mathrm{Hg}$ accumulé dans les mousses aquatiques.
Une étude utilisant des bryophytes terrestres épiphytiques a permis d'exclure une contamination par voie atmosphérique (Roeck 1992).

La nappe phréatique entre le Rhin supérieur et l'Ill dans la zone étudiée présente une sectorisation caractéristique (Fig. 2) avec des secteurs à fortes teneurs en mercure des mousses aquatiques et d'autres à faibles teneurs proches de la teneur de « bruit de fond naturel » de 0,05 $\mu \mathrm{g} \mathrm{Hg} \mathrm{g}^{-1}$ (ms) (Carbiener 1978).

Les sites les plus faiblement contaminés au niveau de la frange rhénane correspondent aux points de prélèvement suivants : $17,21,24,25$. Du côté de l'Ill, ce sont les sites au niveau des deux rivières phréatiques $(7,8$; Tableau 1$)$.

Les sites les plus fortement contaminés sont : la source dans le coude de Rhinau (13), l'Altwasser en amont de sa confluence avec le CCD (20). Le point 20 révélait la teneur moyenne la plus importante de la zone d'étude pour l'année $1991: 0,25(0,14$ à 0,36$)$ $\mu \mathrm{g} \mathrm{Hg} . \mathrm{g}^{-1}$ (ms) ce qui représente une teneur presque identique à celle des échantillons prélevés dans le Rhin à l'aval de l'usine hydroélectrique de Rhinau (1) : $0,24(0,13$ à 0,54$) \mu \mathrm{g} \mathrm{Hg} \cdot \mathrm{g}^{-1} \mathrm{~ms}$ (Tableau 1).

\section{Analyse des secteurs d'infiltration}

L'origine de la sectorisation de la nappe phréatique entre le Rhin et l'Ill réside dans les apports plus ou moins importants de " filtrats rhénans " contaminés par le mercure. Les résultats montrent que ces apports étaient plus importants en 1990, année caractérisée par une forte crue au mois de février et pour laquelle la sectorisation de la nappe est plus contrastée, que pour l'année suivante sans crue importante. Ceci confirme les résultats d'Ubell (1987) qui a observé une augmentation des injections d'eau du Rhin moyen vers la nappe phréatique riveraine pendant et après deux épisodes de crue.

Les apports variables d'eau contaminée du Rhin canalisé vers la nappe phréatique soulèvent la question de savoir quels processus régissent l'injection vers et/ou le drainage de la nappe.

Les facteurs à la base de ces processus relèvent de domaines très différents (la physico-chimie, l'écologie et la physiologie végétale, la dynamique des milieux poreux saturés, l'hydrologie et de la géomorphologie) dont il faut tenir compte pour comprendre les processus générateurs des fonctionnements hydrologiques du Rhin et de l'Ill. 




Fig. 2. Sectorisation de la nappe phréatique en fonction des teneurs en mercure (moyennes annuelles 1990) des mousses aquatiques. iFig. 2. Sectors of the groundwater table as function of $\mathrm{Hg}$ contents (annual means 1990) in the aquatic mosses. 


\begin{tabular}{|c|c|c|c|c|c|c|c|}
\hline & \% & $y_{1}$ & o & ons & row & 4 & \%ox \\
\hline & & wox. & $x^{3}$ & 40 & 30k & knt & 46 \\
\hline & $\overrightarrow{8} \cdot \overrightarrow{8}$ & 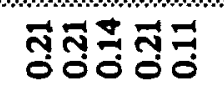 & 80000 & 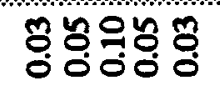 & 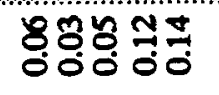 & క్ర & \& \\
\hline & $\frac{\alpha}{d} \cdot \frac{1}{3}$ & 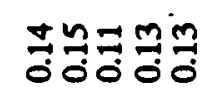 & 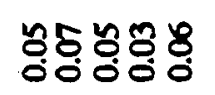 & ธํํㅇํํำำ 궁 & 웅경영경용 & 8영둥융 & ళํํㅇํํำ \\
\hline : & ร్ . & 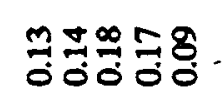 & 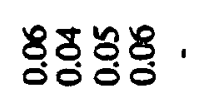 & 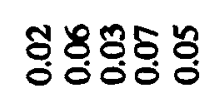 & 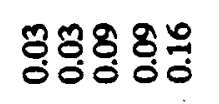 & క్రం: & 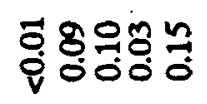 \\
\hline & 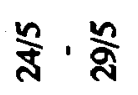 & ํㅜㅇ웅ำํํㅇㅇㅇㅇํ & 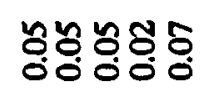 & 융융융용 & 8\%ㅇํㅇำ & \%ి융융ㅇㅇㅇ & 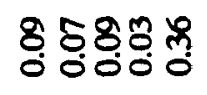 \\
\hline & $\frac{m}{y} \cdot \frac{m}{c}$ & . . F & 8ू. & 둥훙궁응 & 쿵융ำ & 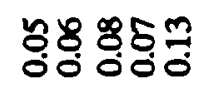 & ํํㅇㅇํㅇ영 \\
\hline & 突. 产 & 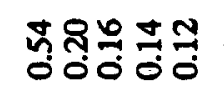 & 궁융융융 & 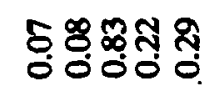 & 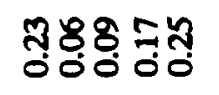 & 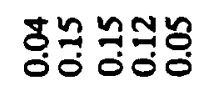 & 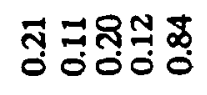 \\
\hline & & 3. & 4 - & 3. & 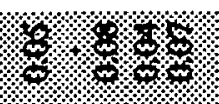 & 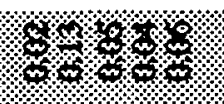 & 680\% \\
\hline & & (3) & 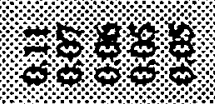 & 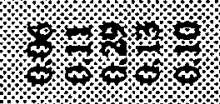 & (2.) & 6 & 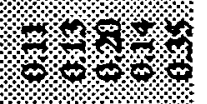 \\
\hline & $\vec{\nabla} \cdot \overrightarrow{\text { Dे }}$ & 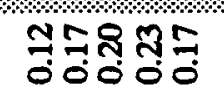 & 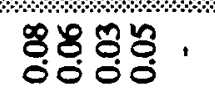 & 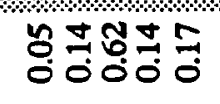 & 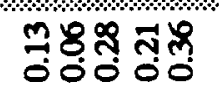 & ठัర & ๑ั 영 స్త \\
\hline & 高. 尔 & สై స్రిశ్రి శ్రీత్ర & 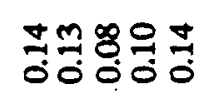 & 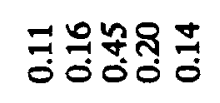 & 공 녕 & 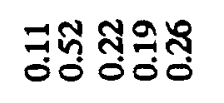 & 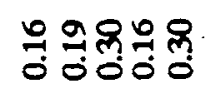 \\
\hline 9 & స. & 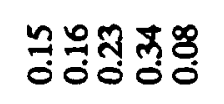 & 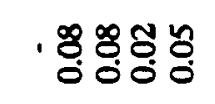 & 형 & ఫ్ర్ర. క్రం: & 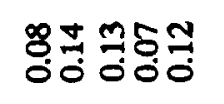 & 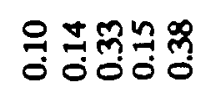 \\
\hline & $\stackrel{n}{\delta} \cdot \frac{n}{m}$ & 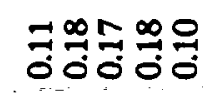 & . & 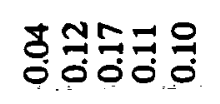 & \% 웅 궁 & 웅궁ำ & 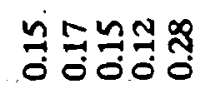 \\
\hline & S. & 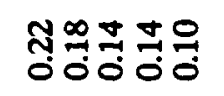 & 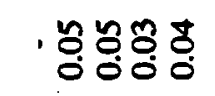 & 뭉융궁융 & 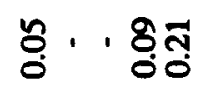 & 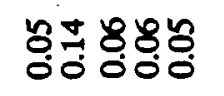 & 웅 8.50 \\
\hline & 곡 & ํㅜㅇㅊㅀㅇ ్ํㅇ궁 & 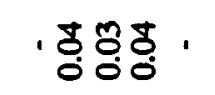 & . \% & $\frac{8}{0} \cdot \cdots \frac{\pi}{0}$ & 8ㄷㅇㅇㅇㅇㅇ & 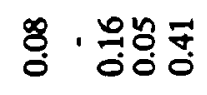 \\
\hline & & & 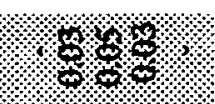 & $8.6 \%=$ & & 6. & 6. \\
\hline & & & $6 \%$ & $4.4 \%$ & & 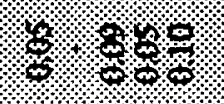 & 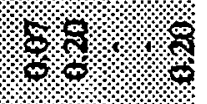 \\
\hline & ำ & 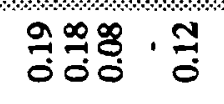 & . & 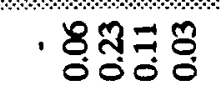 & 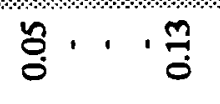 & 홍. & s. \\
\hline & $\stackrel{9}{2}$ & & . & . 콩 궁웅 & $\frac{9}{6} \cdot$ & $\stackrel{8}{0} \cdot \frac{7}{0}=77$ & 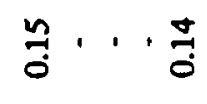 \\
\hline 年 & s. & . . . . & . & 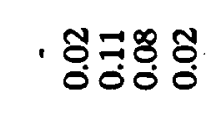 & $\stackrel{0}{0} \cdot$ & శ్ . & $\frac{\delta}{0} \cdot \cdots \frac{a}{0}$ \\
\hline & 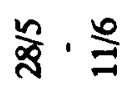 & $\therefore$, 望 & . & 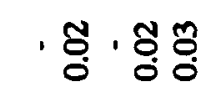 & है. & 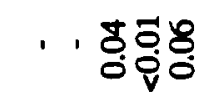 & ర్ \\
\hline & $\frac{m}{d} \cdot \frac{F}{z}$ & . . . & . & 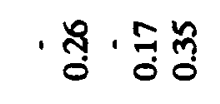 & ㅇ․ & . ' 웅영영 & 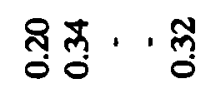 \\
\hline & $\bar{y} \cdot \frac{2}{4}$ & $\cdots, \frac{n}{0}$ & . 8 & $\cdot \frac{ \pm}{0} \cdot, \frac{\tilde{m}}{0}$ & & 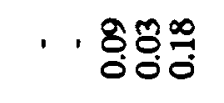 & 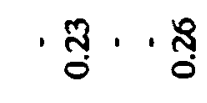 \\
\hline$\underline{E}$ & & $-n$ & & $\exists \cong \cong \pm \cong$ & 노 & シสスホヘ & จละ \\
\hline
\end{tabular}


Les zones les plus fortement contaminées de la frange rhénane sont localisées d'une part au niveau des coudes du Rhin à hauteur de Rhinau (13) et de Dalhunden (28), et d'autre part dans les secteurs situés en amont des retenues d'eau des usines hydroélectriques (Rhinau 22, 23 et Gerstheim 19, 20, 13).

\subsection{Le fonctionnement hydrologique au niveau des coudes du fleuve}

Les mousses aquatiques prélevées dans la source à Rhinau (13, Fig. 1) montrent des teneurs élevées pour toute la période étudiée $(0,29 \mu \mathrm{g}$ en 1990 et 0,24 $\mu \mathrm{g}$ en 1991). Celles-ci sont 5 fois plus importantes que celles du « bruit de fond " de la zone d'étude (rivières phréatiques exemptes de contaminations par le mercure).

Le facteur de contamination FC peut, pour certaines campagnes de prélèvements, atteindre la valeur de $\mathrm{FC}=20$ (Campagne de novembre 1990, Tableau 1) : la teneur de l'échantillon prélevé dans le Bronnwasser (8) se situait à $0,03 \mu \mathrm{g} \mathrm{Hg} . \mathrm{g}^{-1}(\mathrm{~ms})$ tandis que celle de l'échantillon provenant de la source à Rhinau (13) était de $0,62 \mu \mathrm{g} \mathrm{Hg} \cdot \mathrm{g}^{-1}$ (ms).

A la fin du mois de septembre 1990 et à la fin du mois de janvier 1991, les teneurs des mousses prélevées à l'aval de l'usine hydroélectrique de Rhinau (1) sont importantes : 0,28 et $0,54 \mu \mathrm{g} \mathrm{Hg} \cdot \mathrm{g}^{-1}$ (ms) respectivement (Tableau 1). Au cours de l'année 1990, les bryophytes de la source située dans le coude

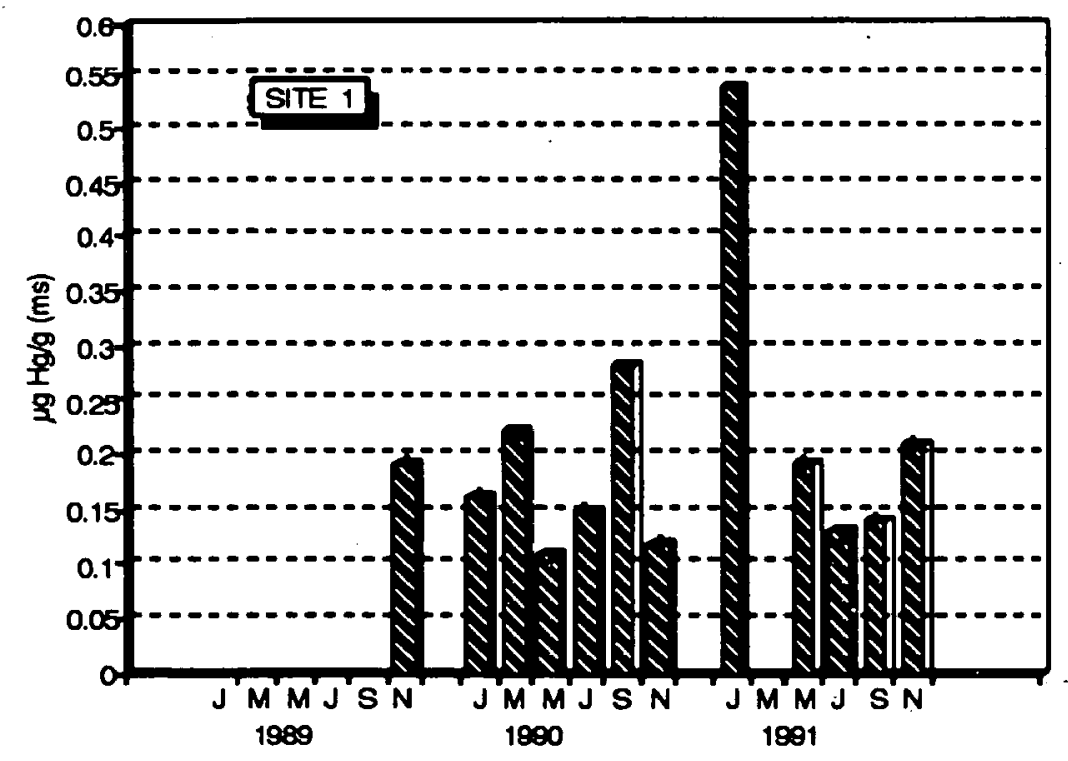

du fleuve atteignent leurs teneurs maximales de toute la période étudiée et dépassent largement celles des mousses prélevées dans le Rhin canalisé (Fig. $3 \mathrm{a}, \mathrm{b}$ ).

Les variations des teneurs en mercure des bryophytes aquatiques d'une source située à $300 \mathrm{~m}$ du fleuve (Fig. 3 b) révèlent une évolution temporelle plus ou moins différée par rapport aux teneurs des mousses prélevées dans le Rhin canalisé (Fig. 3 a). Ce décalage peut être expliqué par un phénomène de retard, l'« hystérésis " : des variations de la physico-chimie de l'eau du Rhin engendrent les mêmes variations de la physico-chimie de l'eau souterraine, mais différée dans le temps (Carbiener \& Trémolières 1990). Les résultats des travaux de Trémolières et al. (1993) sur l'évolution des teneurs en chlorures illustrent bien ce phénomène de l'hystérésis.

Des résultats d'expériences de laboratoire simulant le transport d'ions dans un milieux poreux saturé en colonne indiquent également des phénomènes de retard dans le transport de solutés (Strauss 1991).

L'évolution des teneurs en mercure dans les mousses du Rhin canalisé et de la source pendant la période hivernale 1990/91, ainsi que pour la période autour du 12 mai 1991, ne montre qu'un faible décalage dans le temps; comme si l'eau du fleuve traversait le coude par sous-écoulement rapide (Fig. 3 $a, b)$.

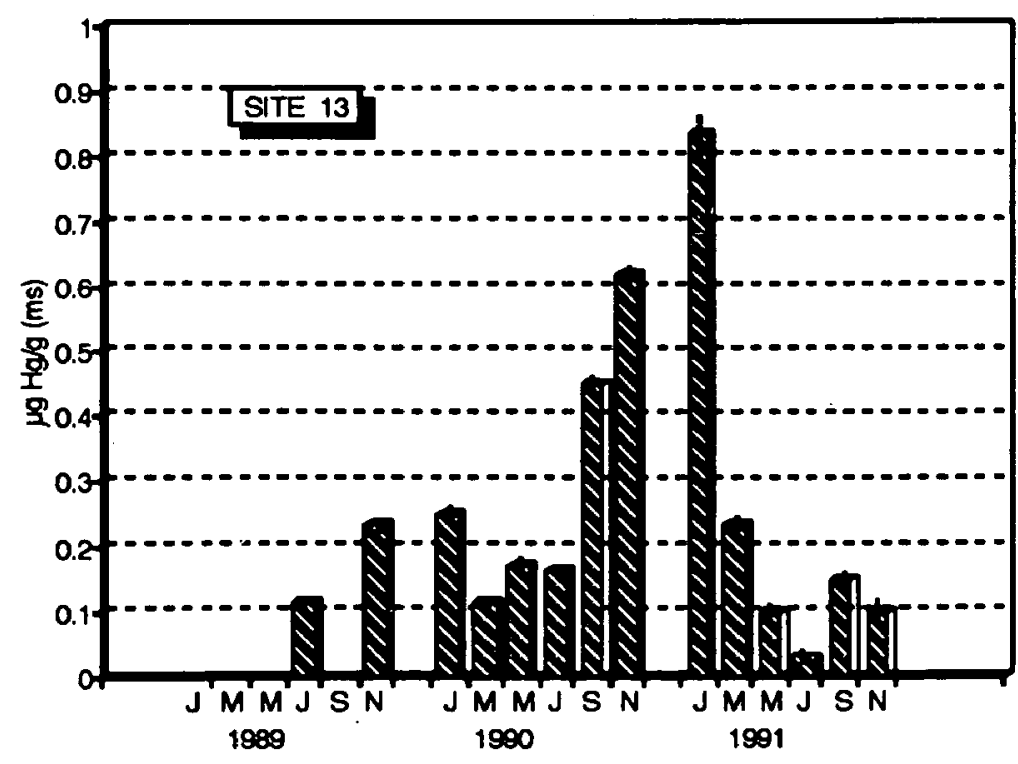

Fig. 3. Evolution des teneurs en $\mathbf{H g}$ des bryophytes aquatiques (1989-1991). - a : Site 1 ; b : Site 13.

Fig. 3. Evolution of the $\mathrm{Hg}$ contents in the the aquatic bryophytes (1989-1991). - a : Site 1 ; b : Site 13. 
L'influence du fleuve sur la nappe phréatique du coude de Rhinau est due à l'importance du volume de « filtrats rhénans » atteignant la nappe phréatique et s'y déplaçant rapidement. Cette zone d'infiltration privilégiée est créée par des paramètres hydrologiques particuliers. Le lit de graviers fonctionnant comme un filtre très grossier, favorise les infiltrations d'eau du Rhin canalisé vers la nappe riveraine, et ceci malgré l'étanchéification des berges lors des travaux de canalisation (EDF 1965).

Au niveau du coude d'un cours d'eau, comme celui de Rhinau ou de Dalhunden, les conditions hydrologiques provoquent des variations de la vitesse du courant le long du profil transversal. La berge concave (rive gauche au niveau du coude de Rhinau) est exposée à un courant fort empêchant les dépôts de sédiments, tandis que le courant du côté de la berge convexe (rive allemande du Rhin à hauteur de Rhinau) est faible favorisant ainsi des dépôts de sédiments. Les échosondages entrepris lors d'une étude réalisée par Roux et al. (1988) au niveau de la berge concave (rive gauche) de l'Ile de Rhinau montre que le lit du Vieux Rhin est érodé sur la rive gauche, tandis que le côté de la rive convexe révèle des dépôts de sédiments importants. De ce fait, le lit graveleux non colmaté du Rhin canalisé au niveau du coude de Rhinau, en tant que prolongement du tracé déjà entamé dans la partie aval du Vieux Rhin de Rhinau, favorise l'injection d'eau du Rhin vers la nappe phréatique et le déplacement rapide des « filtrats rhénans » dans l'aquifère.

Il est probable que les concentrations élevées en mercure de l'eau dans ce secteur de la nappe, et donc des bryophytes aquatiques de la source (13), aient été provoquées par des infiltrations importantes d'eau du fleuve liées à l'augmentation du débit du Rhin à la fin du mois d'octobre et au début du mois de novembre 1990. Le débit avait presque doublé (débit journalier moyen du 30.10.1992: 1200 $\mathrm{m}^{3} . \mathrm{s}^{-1}$ et du $1.11 .1990: 1490 \mathrm{~m}^{3} . \mathrm{s}^{-1}$ ) par rapport à la période d'étiage des 3 mois précédents (août à octobre 1990) au cours de laquelle le débit journalier moyen représentait un volume de $700 \mathrm{~m}^{3} \cdot \mathrm{s}^{-1}$. Dans ce même secteur, des teneurs élevées de mercure ont été trouvées dans les sédiments (Rolland 1991). Le site 13 est également caractérisé par des apports importants en azote ammoniacal et en phosphates en provenance du Rhin canalisé (Eglin \& Robach 1992).

\subsection{Les échanges Rhin-nappe à hauteur des biefs des usines hydroélectriques.}

Le secteur de la nappe phréatique à hauteur du bief de l'usine hydroélectrique de Rhinau est représenté par des points de prélèvement dans le « Contre Canal de Drainage du Rhin " (CCD). Depuis sa source, à hauteur du pk 246 du fleuve (22) et plus vers l'aval $(23,24)$, ce dernier révèle des contaminations mercurielles très importantes atteignant leur maximum en septembre 1990 (Tableau 1). Les sites 23 et 24 se situent à l'aval d'affluents, drains de la nappe phréatique occidentale, le Steingriengiessen et le Muhlbach de Schoenau en amont du site 23 et l'Ischert en amont immédiat du point de prélèvement 24 (Fig. 1). Ces cours d'eau sont épurés lors de la traversée d'un important massif forestier (Trémolières et al. 1993). Il s'agit d'un effet de dilution par des apports de la nappe occidentale peu contaminée.

Les résultats indiquent que l'augmentation des débits du Rhin $\left(510 \mathrm{~m}^{3} . \mathrm{s}^{-1}\right.$ le $21.9 ., 1230 \mathrm{~m}^{3} . \mathrm{s}^{-1}$ le 24.9.1990) provoque, comme au niveau des coudes, des infiltrations intenses d'eau coṇtaminée du fleuve canalisé vers sa nappe riveraine.

Le Rhin canalisé au niveau des biefs surplombe sa plaine d'au moins 10 mètres et le lit du fleuve montre un degré de colmatage très variable. La colonne d'eau exerce une pression hydrostatique importante sur'le lit de gravier peu colmaté, donc très perméable. Une colonne d'eau de $10 \mathrm{~m}$, par exemple, exerce une pression de $1 \mathrm{~kg} . \mathrm{cm}^{-2}$. Cette dernière croît proportionnellement avec l'augmentation du niveau d'eau dans le bief. Il est de ce fait envisageable que les infiltrations dues au débit augmenté du Rhin aient entraîné des teneurs plus élevées dans les mousses aquatiques prélevées peu de temps après dans le cours du CCD, aux points de prélèvement $22\left(0,52 \mu \mathrm{g} \mathrm{Hg}^{\mathrm{kg}} \mathrm{kg}^{-1}\right.$, le 28.9), $23(0,22$ $\mu \mathrm{g} \mathrm{Hg} . \mathrm{kg}^{-1}$, le 28.9$)$ et $24\left(0,19 \mu \mathrm{g} \mathrm{Hg} \cdot \mathrm{kg}^{-1}\right.$, le 3.10).

La distribution amont-aval des teneurs en mercure dans ce secteur de la nappe rhénane révèle la cinétique de la propagation du contaminant $\mathrm{Hg}$ dans la nappe. Elle illustre l'influence des affluents phréatiques d'une part (effet de dilution) et celle du degré de colmatage du lit du Rhin canalisé d'autre part (diminution des infiltrations avec augmentation du colmatage).

En effet, les teneurs en mercure relevées au site 24 , situé à seulement $150 \mathrm{~m}$ à l'amont du barrage, 
où tendent à s'accumuler le plus de sédiments (Ramade 1981), sont les plus faibles du secteur : 15 prélèvements sur 18 révèlent des teneurs inférieures à $0,10 \mu \mathrm{g} \mathrm{Hg} \cdot \mathrm{kg}^{-1}$ (ms) (Tableau 1).

Dans la partie amont du barrage, le Rhin canalisé comprend un lit bétonné, sur une longueur de $30 \mathrm{~m}$ (EDF 1965). De plus, la partie du lit de graviers du bief situé à proximité immédiate du barrage de l'usine hydroélectrique montre un degré de colmatage plus important que celui dans la partie amont du bief de l'île de Rhinau, à hauteur du barrage de la prise d'eau du Vieux Rhin (EDF 1965). De ce fait, la portion du lit colmaté à proximité de l'usine hydroélectrique fonctionne comme un écran d'étanchéité qui empêche les infiltrations d'eau du bief vers cette zone de la nappe riveraine.

A hauteur du bief de Gerstheim (Fig. 1), le réseau de points de prélèvement comprend deux sites dans le CCD $(18,13)$ et plusieurs points de prélèvement dans deux anciens bras du Rhin déconnectés du fleuve depuis la canalisation de ce dernier : le Brunnenwasser de Rhinau-Daubensand $(14,15,16,17)$ et l'Altwasser de Daubensand $(19,20)$ (Fig. 1). On retrouve au niveau de ce bief le même schéma de fonctionnement que celui à hauteur du bief de Rhinau.

La zone la plus fortement contaminée dans ce secteur correspond au cours aval de l'Altwasser (20), zone d'injection d'eau du Rhin, également décelée par des apports importants d'azote ammoniacal $\left(19,0\right.$ à $880 \mu \mathrm{g} . \mathrm{I}^{-1} \mathrm{~N}^{-\mathrm{NH}_{4}}{ }^{+}$contribuant à l'installation d'associations végétales aquatiques eutrophes (Eglin \& Robach 1992) et par les fortes teneurs en mercure des sédiments (Rolland 1991).

Les mousses du secteur amont de la rivière Altwasser (18) alimentée par les eaux du CCD révèlent des teneurs en mercure variables, mais peu élevées (Tableau 1). Le long du cours de cette rivière, les teneurs en mercure augmentent régulièrement pour atteindre la valeur maximum dans la partie aval (20).

Les résultats montrent que les cours moyens et aval de cette rivière phréatique subissent une contamination généralisée, qui varie peu dans le temps (Tableau 1), par des «filtrats rhénans » contaminés et confirment ainsi les résultats du BRGM/SGAL (1978), Roeck et al. (1991), Rolland (1991) et Eglin \& Robach (1992). Au niveau de cette zone d'infiltration intense, les « filtrats rhénans" semblent emprunter le pavage de l'ancien tracé de
l'Altwasser, un bras de type torrentiel avant la canalisation, sous forme de sous-écoulements importants franchissant les berges à partir d'une dépression dans le lit de gravier du fleuve canalisé au pk 266 (EDF 1965). Suite au remaniement des matériaux lors des travaux de canalisation du Rhin comprenant le compactage et l'étanchéification des berges (elles sont bétonnées) les eaux du Rhin s'infiltrent par le lit du fleuve et viennent ainsi contaminer des secteurs phréatiques plus éloignés du fleuve. Ceci expliquerait pourquoi les teneurs (moyennes en mercure 1990 et 1991) dans les mousses aquatiques prélevées dans le CCD sont généralement inférieures (sites 18 avec 0,15 et $0,08 \mu \mathrm{g} \mathrm{Hg} . \mathrm{g}^{-1}$ (ms) et 27 avec 0,13 et $0,10 \mu \mathrm{g} \mathrm{Hg} \cdot \mathrm{g}^{-1}(\mathrm{~ms})$ à celles des sites plus éloignés du fleuve $\left(19: 0,14\right.$ et $0,13 \mu \mathrm{g} \mathrm{Hg} . \mathrm{g}^{-1}$ (ms), $20: 0,25 \mu \mathrm{g} \mathrm{Hg} \cdot \mathrm{g}^{-1}$ (ms), en 1990 et 1991.

Mais globalement, la baisse des teneurs moyennes en mercure le long d'un transect est-ouest, entre le Rhin canalisé et l'Ill, révèle que l'influence rhénane sur la nappe phréatique diminue, au fur et à mesure qu'on s'éloigne du fleuve. Les teneurs moyennes en mercure atteignent, avec l'éloignement du Rhin canalisé, des teneurs proches du « bruit de fond naturel » des bryophytes aquatiques (Fig. 4).

Le transect ainsi réalisé révèle un gradient de concentration en mercure dans l'eau souterraine :

- des concentrations importantes en mercure caractérisent la nappe phréatique à proximité des biefs $(20,22)$;

- des concentrations de plus en plus faibles apparaissent avec l'éloignement des sites par rapport au fleuve (site 16) ;

- à $3 \mathrm{~km}$ environ du fleuve (site 9), l'influence du Rhin semble disparaître.

\section{Les secteurs de drainage}

\subsection{Le secteur rhénan entre Diebolsheim et Rhinau}

Trois sites, $(21,24,25)$ suivis au niveau de la frange rhénane se distinguent par leurs faibles teneurs moyennes en mercure (Tableau 1, Fig. 1). Les teneurs généralement faibles en mercure démontrent que les infiltrations d'eau à partir du fleuve canalisé vers la nappe riveraine sont faibles ou presque inexistantes.

Le niveau du Rhin canalisé dans ce secteur correspond à celui du canal de fuite de l'usine hydroélectrique. 


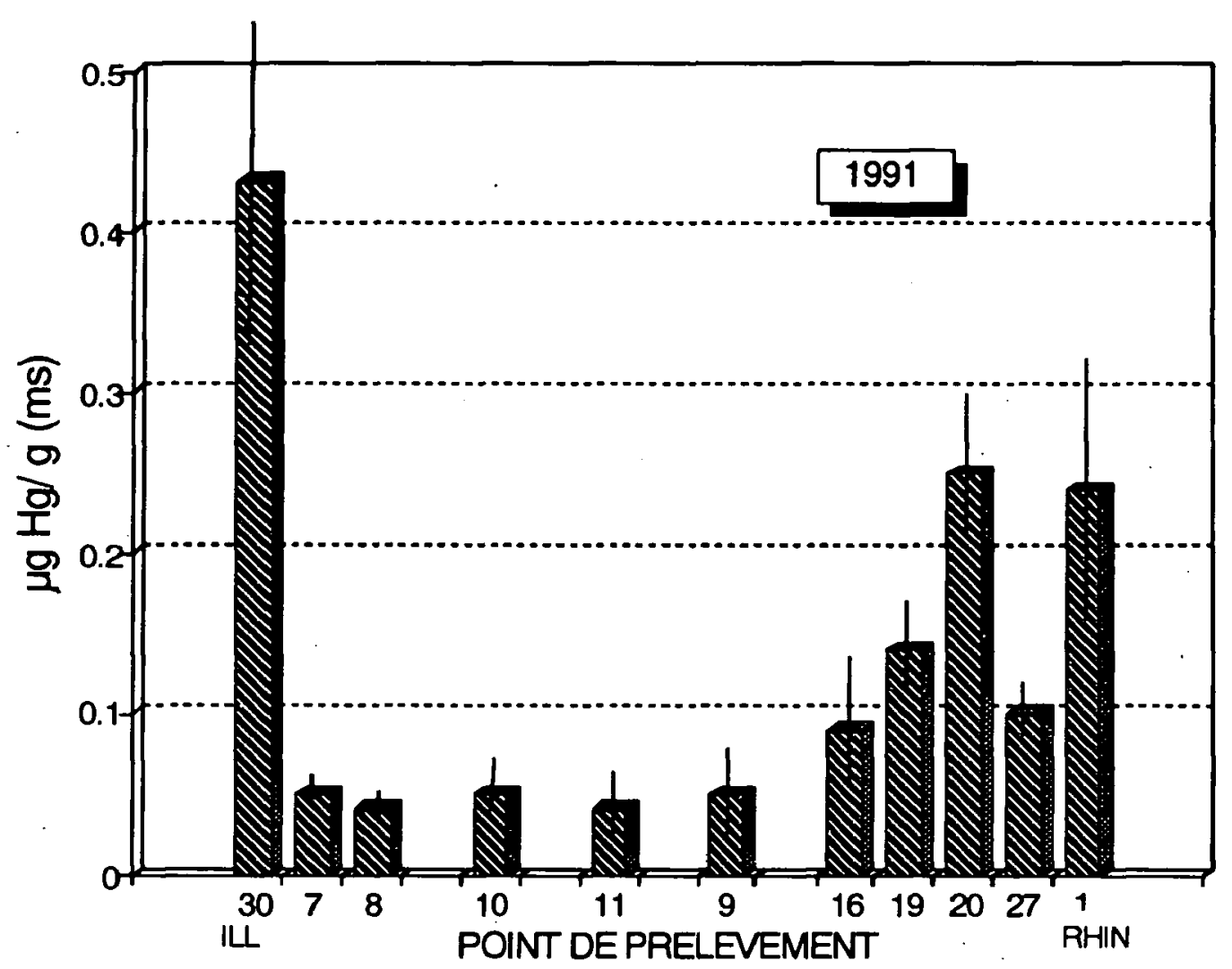

Fig. 4. Teneurs en mercure (moyennes annuelles 1991) des mousses aquatiques le long d'un transect entre l'Ill et le Rhin canalisé. Fig. 4. Mercury contents (annual means 1991) in the aquatic mosses following a transect between the Ill and the canalized Rhine.

Les observations recueillies au niveau d'une buse reliant le canal de fuite et le CCD montrent que le niveau du canal de fuite se situait pendant presque toute la période étudiée en-dessous du niveau de la nappe phréatique. En effet, le canal de fuite fonctionne comme drain de cette dernière, à l'exception de la période de crue en février 1990 qui a provoqué une teneur moyenne annuelle légèrement plus élevée au point de prélèvement 25 (Tableau 1).

Lors de la crue, de l'eau du Rhin passait directement dans le CCD. Pendant le reste de la période d'étude, l'écoulement de l'eau s'effectuait à partir du CCD vers le canal de fuite. Les teneurs faibles en mercure révélées aux trois sites dans ce secteur confirment l'action drainante du Rhin sur sa nappe dans la zone aval de l'usine EDF.

Les résultats montrent également, que l'effet de drainage apparaît déjà dans la zone située dans le CCD à hauteur du barrage hydroélectrique représentée par le point de prélèvement 24 . Ici les teneurs se rapprochent, surtout en 1991, année sans crue importante, de la teneur de sites exempts de pollution par le mercure $(7,8,9,10,11$ : Tableau 1).

\subsection{Le secteur de l'Ill à l'aval de Benfeld}

En examinant les teneurs en mercure des bryophytes prélevés dans l'Ill à Benfeld (30) et dans les rivières phréatiques riveraines représentées par la Lutter à Benfeld (7) et le Bronnwasser à Osthouse (8), nous constatons que les teneurs moyennes en mercure dans les rivières phréatiques sont très faibles, malgré leur proximité par rapport à l'Ill (300 et $800 \mathrm{~m}$ respectivement), tandis que le site de l'Ill se révèle fortement contaminé par ce métal (Fig. 4). Les teneurs moyennes en mercure des mousses prélevées dans les rivières phréatiques ellanes sont proches de celles dues au « bruit de fond » de la zone d'étude et indiquent l'action drainante de l'Ill sur sa nappe phréatique à hauteur et à l'aval de Benfeld (Fig. 5).

Cette rivière, déconnectée du Rhin depuis la période postglaciaire, coule dans la dépression occidentale marginale de la plaine. Le niveau de l'eau dans l'Ill se trouve en-dessous du niveau de la nappe phréatique, surtout en période d'étiage. De plus, son lit sablo-limoneux montre un degré de colmatage plus important que celui du Rhin. 




Fig. 5. Déplacement de l'eau souterraine entre l'Ill et le Rhin à hauteur de l'axe Benfeld-Rhinau.

Fig. 5. Groundwater flow in the sector between the Ill river at Benfeld and the canalized Rhine at Rhinau. 
Les résultats obtenus à l'aide des mousses aquatiques révèlent la qualité excellente de l'eau souterraine de ce secteur vis-à-vis du $\mathrm{Hg}$, mais aussi vis-àvis des phosphates et de l'azote ammoniacal, résultats présentés dans les travaux de Klein \& Carbiener (1988) utilisant l'échelle de bioindication phytosociologique.

La capacité d'épuration de l'eau de crue au niveau du champ d'inondation de l'Ill réside d'une part dans la capacité d'échange de cations des sols argilosablo-limoneux et, d'autre part, dans la capacité épuratrice du système « sol-végétation » particulièrement efficace vis-à-vis des eutrophisants (Trémolières et al. 1991, Sanchez-Perez 1992).

\section{Conclusion}

Le Rhin canalisé contamine sa nappe alluviale riveraine par des infiltrations appelées « filtrats rhénans » (Carbiener et al. 1988, Carbiener \& Trémolières 1990, Trémolières et al. 1993).

La suppression des surfaces inondables et la création de biefs surplombant la plaine lors de la canalisation du Rhin supérieur ont accentué l'action contaminante du fleuve sur sa nappe phréatique riveraine : l'eau du Rhin n'est plus épurée au niveau d'un champ d'inondation fonctionnel excepté au niveau des îles du Rhin restées inondables (Carbiener \& Herrscher 1989, Sanchez-Perez 1992). De ce fait, toutes les rivières phréatiques sous influence rhénane sont eutrophes et contaminées par des quantités variables d'eutrophisants (phosphates, azote) et de polluants persistants, comme le mercure. Ces polluants créent ainsi une pollution chronique de l'eau souterraine (Carbiener \& Trémolières 1990, Eglin \& Robach 1992).

Les infiltrations d'eau du Rhin vers la nappe sont favorisées dans les secteurs amont des usines hydroélectriques et dans les coudes du fleuve. La perméabilité du lit graveleux, peu colmaté, du Rhin canalisé associé à la pression hydrostatique excercée par la colonne d'eau au niveau des bassins de rétention représente le facteur principal responsable de i) la grande vulnérabilité de la nappe phréatique dans ces secteurs et de ii) la contamination de ces secteurs de la nappe par les "filtrats rhénans ". Seul un secteur, situé à l'aval du barrage de l'usine hydroélectrique de Rhinau, au niveau du canal de fuite, correspond à un secteur de drainage de la nappe alluviale par le Rhin. Dans ce secteur, le niveau du Rhin canalisé est inférieur à celui de la nappe phréatique riveraine ce qui crée des conditions hydrodynamiques provoquant le drainage de la frange superficielle de la nappe ainsi qu'un appel d'eau souterraine très pure du secteur central de la plaine.

Les résultats de ce travail ont permis de confirmer l'impact négatif des aménagements du Rhin sur la qualité de l'eau souterraine dans la plaine d'Alsace. En effet, une pollution accidentelle ou chronique importante du Rhin supérieur risque de contaminer considérablement la nappe phréatique, surtout au niveau des zones d'infiltration importante (Fig. 5.).

En revanche, malgré la forte pollution de l'Ill par le mercure (actuellement plus forte que celle du Rhin), la qualité de l'eau des rivières phréatiques ellanes, à hauteur et à l'aval de Benfeld, reste très bonne avec des faibles teneurs en mercure, en phosphore et en azote ammoniacal (Trémolières et al. 1993, Roeck 1992). D'une part, l'Ill dans ce secteur ne fuit pas dans son lit, d'autre part, le champ d'inondation fonctionnel de l'Ill joue ici un rôle très important dans la recharge de la nappe phréatique en eau de très bonne qualité : il assure l'épuration des eaux de crue lors de leur infiltration.

Un aménagement de l'Ill (canalisation supprimant le champ inondable et augmentant le niveau de l'eau dans la rivière) aurait les mêmes conséquences que celles observées dans le secteur rhénan.

\section{Remerciements}

Cette étude a été réalisée au Laboratoire de Botanique et d'Ecologie végétale (URA 95 CEREG, Département des Sciences de l'Environnement, Faculté de Pharmacie) avec le soutien financier du PIREN-EAU/Alsace, de la Fondation Sandoz pour le Rhin et du Laboratoire d'Hydrologie de l'Université Louis Pasteur de Strasbourg.

\section{Travaux cités}

Amoros C., Bravard J.P., Reygrobellet J.L., Pautou G. \& Roux A.L. 1988. - Les concepts d'hydrosystème et de secteur fonctionnel dans l'analyse des systèmes fluviaux à l'échelle des écocomplexes. Bull. Ecol., 19 : 531-546.

BRGM-SGAL 1978. - Etude de l'invasion des eaux rhénanes dans la nappe phréatique d'Alsace en aval de Fessenheim. Rapport BRGM-SGAL 0133, Ed. SRAE, Horburg-Wihr : 29 p.

Carbiener R. 1977. - Serious Mercury Pollution of the Rhine and its Tributaries in Alsace proved by Ecological Evidence : The role of Fish and Birds of prey as Detectors. Vegetation Science and Environmental Protection. Proceedings of The International Symposium in Tokyo on Protection of the Environment and Excursion on Vegetation Science Through Japan : June 5-7, 1974 (Edit. : A. Iyawaki \& R. Tüxen, with collab. of S. Okuda), Maruzen Co., LTD., Tokyo : 201-206. 
Carbiener R. 1978. - Etude écologique de la pollution par le mercure du bassin du Rhin en Alsace et de son évolution. 1973-1977. Etablissement Public Régional Université Louis Pasteur, Région Alsace, Service régional d'Aménagement des eaux, Strasbourg et Colmar : 149 p.

Carbiener R. 1983. - Le Grand Ried Central d'Alsace : écologie et évolution d'une zone humide d'origine fluviale rhénane. Bull. Ecol. 14 (4) : 249-277.

Carbiener R. \& Herrscher P. 1989. - Le Grand Ried Central d'Alsace : Ecologie d'un paysage. Nos Rieds, demain..., Bull. Soc. Ind. Mulh. $813: 23-59$.

Carbiener R. \& Ortscheit A. 1987. - Wasserpflanzengesellschaften als Hilfe zur Qualitätsüberwachung eines der grössten Grundwasser-Vorkommens Europas (Oberrheinebene) dans : Miyawaki A. et al., Vegetation Ecology and Creation of New Environments. Proceed. Intern. Symp. Tokyo... 1987, Tokai Univ. Press Tokyo 160, Japan : 283-312.

Carbiener R., Trémolières M., Ortscheit A. \& Klein J.P. 1988. - Associations végétales biorévélatrices des échanges hydrologiques « eaux de surface-eaux souterraines ». Coll. franco-allemand, Contamination des eaux souterraines par les nitrates, incidences de l'agriculture sur la qualité des eaux souterraines et mesures de protection. 6 octobre 1988, Univ.Stuttgart et ULP Strasbourg, cahier $71: 171-200$.

Carbiener R. \& Trémolières M. 1990. - The Rhine rift valley groundwater - river interactions : evolution of their susceptibility to pollution. Regulated Rivers : Research \& Water Management 5 : 375-389.

Castella E. \& Amoros C. 1986. - Diagnostic phyto-écologique sur les anciens méandres dans : Recherches interdisciplinaires sur les écosystèmes de la basse plaine de l'Ain (France) : potentialités évolutives et gestion. Doc. Cartogr. Ecologique 29 : 97-108.

Cloots-Hirsch A.R. 1987. - Echanges nappe-rivières et genèse des inondations dans le Ried Central de l'Ill (Alsace) : Premiers résultats. Crues et inondations (Genèse, Méthodes d'Etude, Impacts et Prévision). Actes du Colloque de Strasbourg : 16-18 octobre 1986, édité par : C.E.R.E.G., Université Louis Pasteur Strasbourg : 47-60.

Davies B.R., Thomas M. \& Meador M. 1992. - An assessment of the ecological impact of inter-basin water transfers and their threats to river basin integrity and conservation. Aquatic Conserv. : Mar. \& Freshw. Ecosyst. 2 : 325-349.

Electricité de France (EDF) 1965. - Aménagement du Rhin. $7^{e}$ Bief. Chute de Gerstheim. Plan du barrage $(1: 200,1$ : 2000).

Eglin I. \& Robach F. 1992. - Typologie et végétation de l'hydrosystème rhénan dans le secteur central de la plaine d'Alsace : Interprétation et fonctionnement écologique. I. Unités fonctionnelles connectées au Rhin. II. Unités fonctionnelles déconnectées du Rhin. Thèse de doctorat de l'Université Louis Pasteur, Strasbourg : 342 p + annexes.

Förstner U. \& Wittmann G.T.W. 1981. - Metal Pollution in the Aquatic Environment. Springer Verlag, Berlin : 486 p.

Haslam S.M. 1992. - River Pollution : An Ecological Perspective. Belhaven Press, London, New York : $253 \mathrm{p}$.

Klein J.P. 1988. - Approche phytosociologique du fonctionnement hydrologique de deux rivières phréatiques : la Lutter et le Bronnwasser, Mémoire de D.E.A. Université Louis Pasteur, Strasbourg : $48 \mathrm{p}$.
Klein J.P. \& Carbiener R. 1988. - Effets de crues de l'Ill sur les phytocénoses aquatiques de deux rivières phréatiques du secteur de Benfeld et d'Erstein : la Lutter et le Bronnwasser. Intérêt des plantes aquatiques comme bioindicateurs d'eutrophisation. Bull. Ass. Phil. Als. Lor. 24 : 3-34.

Krause W. \& Carbiener R. 1975. - Die Chloridkonzentration in den Gewässern des Oberrheinebene und ihrer Randgebiete. Erdkunde, Archiv für wissenschaftliche Geographie, 29 (4) : 267-277.

Lafont M. \& Durbec A. 1990 . - Essai de description biologique des interactions entre eau de surface et eau souterraine : vulnérabilité d'un aquifère à la pollution d'un fleuve. Annls Limnol. 26 (2) : 119-129.

Mouvet C. 1986 . - Métaux lourds et mousses aquatiques. Synthèse méthodologique. Laboratoire d'écologie. Université de Metz. Agence de l'Eau Rhin-Meuse. Agence RhôneMéditerranée-Corse : $74 \mathrm{p}+$ annexes.

Ramade F. 1981. - Ecologie des ressources naturelles. Ecologie appliquée et sciences de l'environnement 4, Masson, Paris : $322 \mathrm{p}$.

Robach F., Eglin I. \& Carbiener R. 1991. - Hydrosystème rhénan - Evolution parallèle de la végétation aquatique et de la qualité de l'eau (Rhinau). Bull. Ecol. 22 (1) : 227-241.

Roeck U. 1989. - Utilisation de bioindicateurs bryophytes pour l'étude comparative des échanges rivières (Rhin, Ill)-nappe phréatique en Plaine d'Alsace. Mémoire de DEA « Systèmes spatiaux et aménagements régionaux », Université Louis Pasteur, Strasbourg : $117 \mathrm{p}$.

Roeck U. 1992. - Le transfert du mercure $(\mathrm{Hg})$ utilisé comme descripteur du fonctionnement hydrologique (échanges cours d'eau-nappe) dans la plaine du Rhin supérieur en Alsace. Thèse de Doctorat. Université Louis Pasteur, Strasbourg : $195 \mathrm{p}+$ annexes.

Roeck U., Trémolières M., Exinger A. \& Carbiener R. 1991. Les mousses aquatiques, bioindicateurs du niveau de pollution chimique. Utilisation des mousses aquatiques dans une étude sur le transfert du mercure en tant que descripteur du fonctionnement hydrologique (échanges cours d'eau-nappe) en plaine d'Alsace. Hydoécol. Appl. 3 (2) : 241-256.

Rolland T. 1991. - Le transfert du mercure dans des écosystèmes riverains en tant que témoin des échanges fleuve-nappe en amont de Strasbourg. Mémoire de DEA « Toxicologie de l'environnement ». Université Louis Pasteur, Strasbourg et Université de Metz : $28 \mathrm{p}+$ annexes.

Roux A.L. 1988. - Analyse de l'état actuel des écosystèmes aquatiques rhénans et suivi de leur reconstitution durant trois années dans le secteur de Rhinau. Contrat CNRS 8202662 : $7 \mathrm{p}$.

Sanchez-Perez J.M. 1992. - Fonctionnement hydrochimique d'un écosystème de la plaine du Rhin : La forêt alluviale du secteur de l'île de Rhinau en Alsace. Thèse de Doctorat de l'Université Louis Pasteur, Strasbourg : $166 \mathrm{p}+$ annexes.

Simler L., Valentin J. \& Duprat A. 1979. - La nappe phréatique de la plaine du Rhin en Alsace. Sciences géologiques, 60 : $266 \mathrm{p}$.

Smith A.J.E. 1980. - The Moss Flora of Britain and Ireland. Cambridge University Press. Cambridge, London : 706 p. 
Strauss J.M. 1991. - Modélisation des interactions physicochimiques à l'interface liquide/solide pendant le transport d'une solution de Mercure II dans un milieu poreux naturel saturé. Thèse de Doctorat. Université Louis Pasteur. Strasbourg : $111 \mathrm{p}$.

Trémolières M., Carbiener D., Carbiener R., Eglin I., Robach F., Sanchez-Perez J.M., Schnitzler A. \& Weiss D. 1991.

Zones inondables, végétation et qualité de l'eau en milieu alluvial rhénan : L'île de Rhinau, un site de recherches intégrées. Bull. Ecol. 22 (3-4) : 317-336.
Trémolières M., Eglin I., Roeck U. \& Carbiener R. 1993. The exchange between river and groundwater in central Alsace (Eastern France) : The' use of aquatic macrophyte communities as ecological descriptors. I. the case of the canalised river Rhine. Hydrobiologia, 254 : 133-148.

Ubell K. 1987. - Austauschvorgänge zwischen Fluss- und Grundwasser - Teil 1, Deut. Gewässerkundl. Mitt. 31 (4), Bundesanstalt für Gewässerkunde, Koblenz : 119-126. 